\title{
Missile Position Tracking using Kalman Filter
}

\author{
Yassir Obeid Mohammed ${ }^{1}$, Dr. Abdelrasoul Jabar Alzubaidi ${ }^{2}$ \\ ${ }^{1}$ Sudan Academy of Sciences (SAS); Council of Engineering Researches \& Industrial Technologies, \\ ${ }^{2}$ Electronics Dept-Engineering College -Sudan University of science and Technology
}

\begin{abstract}
The main element in any missile tracking system is the dynamic state estimator. kalman filter is the most popular estimate approach to recursive linear estimation. This paper proposes and investigates the use of this method in missile tracking system.
\end{abstract}

Keywords: - missile, tracking, position, estimation, kalman filter, Gaussian, Algorithm.

\section{INTRODUCTION}

Many problems in science require estimation of the state of a system or an element in the system that changes over time using a sequence of noisy measurements made on the system[2]. Modeling of such system focuses on the state vector of the system. The state vector contains all relevant information required to describe the system under investigation. For example, in tracking problems, this information could be related to the kinematic characteristics of the target. In this work the extended Kalman filter is used to estimate the position of an attacking missile to predict its location and hence prevent it from hitting its target in next step. A simulation is used to achieve results.

\section{KALMAN FILTER IMPLEMENTATION:}

The kalman filter is an algorithm which uses a series of measurements observed over time, containing noise (random variations) and other inaccuracies, and produces estimates of unknown variables that tend to be more precise than those that would be based on a single measurement alone [3]

Kalman filter addresses the general problem of trying to estimate the state of a discrete-time controlled process [4]. The Kalman filter model assumes the true state at time $\mathrm{k}$ is evolved from the state at $(\mathrm{k}-1)$ according to $\mathbf{x}_{k}=\mathbf{F}_{k} \mathbf{x}_{k-1}+\mathbf{B}_{k} \mathbf{u}_{k}+\mathbf{w}_{k}$ where

- $\quad \mathbf{F}_{k}$ is the state transition model which is applied to the previous state $\mathbf{x}_{k-1} ;$
- $\quad \mathbf{B}_{k}$ is the control-input model which is applied to the control vector $\mathbf{u}_{k} ;$
$\mathbf{w}_{k}$ is the process noise which is assumed to be drawn from a zero mean multivariate normal distribution with covariance $\mathrm{Q}_{\mathrm{k}}$.

$\mathbf{w}_{k} \sim N\left(0, \mathbf{Q}_{k}\right)$

At time $k$ an observation (or measurement) $\mathbf{z}_{k}$ of the true state $\mathbf{x}_{k}$ is made according to

$\mathbf{z}_{k}=\mathbf{H}_{k} \mathbf{x}_{k}+\mathbf{v}_{k}$

where $\mathbf{H}_{k}$ is the observation model which maps the true state space into the observed space and $\mathbf{v}_{k}$ is the observation noise which is assumed to be zero mean Gaussian white noise with covariance $\mathbf{R}_{k}$.

$\mathbf{v}_{k} \sim N\left(0, \mathbf{R}_{k}\right)$

The initial state, and the noise vectors at each step $\left\{\mathbf{x}_{0}, \mathbf{w}_{1}, \ldots, \mathbf{w}_{k}, \mathbf{v}_{1} \ldots \mathbf{v}_{k}\right\}$ are all assumed to be mutually independent. In practice, their matrices might change with each time step or measurement, however sometimes, as it is considered in this case, they are assumed to be constant.

Consider a missile falling towards a target with a constant gravitational field. Let $y(t)$ denote the height of the missile, then

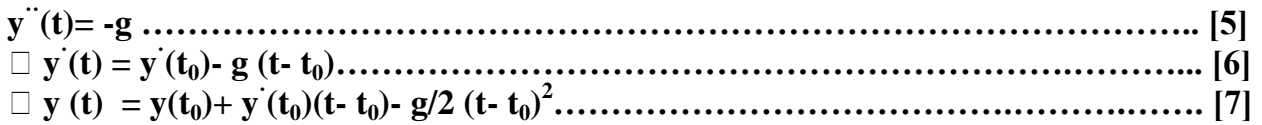

As a discrete time system with time increment of $t-t 0=1$

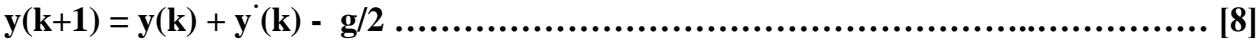

the height $\mathrm{y}(\mathrm{k}+1)$ depends on the previous velocity and height at time $\mathrm{k}$. the state can be define as:

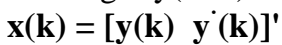

and then the state equation becomes

$y(k+1)=\left[\begin{array}{ll}1 & 1 \\ 0 & 1\end{array}\right] x(k)+\begin{gathered}0.5 \\ 1\end{gathered}(-g)$ 
$=\mathbf{F} \mathbf{x}(\mathbf{k})+\mathbf{G u}$

[11]

Assuming the height of the missile is observed or measured directly. The measurement equation is:

$\mathbf{Z}(\mathbf{k})=\left[\begin{array}{ll}1 & 0\end{array}\right] \mathbf{x}(\mathbf{k})+\mathbf{w}(\mathbf{k})$

$=\mathbf{H} \mathbf{x}(\mathbf{k})+w(\mathbf{k})$

[13]

The variance of $w(k)$ needs to be known for implementing a Kalman filter.

Given the initial state and covariance, we have sufficient information to find the optimal state estimate using the Kalman filter equations.

Assuming an initial true state of position $=100$ and velocity $=0, \mathrm{~g}=1$. an initial estimate state estimate $\mathrm{x}(0)$ and initial state covariance $\mathrm{P}(0)$ are chosen based on mainly intuition. The state noise covariance is all zeros. The measurement noise covariance is estimated from knowledge of predicted observation errors, chosen as 1 here.

\section{APPROACH}

The work method can be summarized in the following two phases.

1) Time Update ("Predict")

1. Project the state ahead according to the equation:

2. Project the error covariance ahead

$$
\mathbf{x}(\mathbf{k})=\left[\mathbf{y}(\mathbf{k}) \mathbf{y}^{\prime}(\mathbf{k})\right]^{\prime}
$$

2) Measurement Update ("Correct")

1. Compute the Kalman gain

2. Update estimate with measurement $\mathrm{zk}$

3. Update the error covariance

\section{ALGORITHM}

The proposed computer algorithm based on Kalman filter approach and Radar detection is:

Start

--- generate a hypothetical target .

$\mathrm{km} / \mathrm{hr}$ ).

(azimuth $=45$ degrees, range $=200 \mathrm{~km}$., heading $=90$ degrees east, speed $=800$

--- generate a tracker aircraft ( or missile).

(azimuth $=45$ digress, range $=70 \mathrm{~km}$., heading $=90$ degrees east, speed $=1200 \mathrm{~km} / \mathrm{hr}$ ).

--- assume that both the target and the tracker are detected by Radar.

--- apply Kalman filter prediction formula to give the estimated target position.

--- maneuver the heading of the tracker based on the estimated coordinates of the target.

--- if the distance between the target and the tracker approaches zero is true.

then collision is assumed.

otherwise the target and the tracker are in visual contact.

end

\section{RESULTS}

Considering assumptions made above and Since values of F, G, H are known the Kalman filter equations can be applied. Results o the application of these equations is summarized in table 1.

Table 1: results

\begin{tabular}{|c|c|c|c|c|c|c|c|}
\hline & \multicolumn{2}{|c|}{ True Values } & \multicolumn{3}{|c|}{\begin{tabular}{l|l} 
& Estimates
\end{tabular}} & \multicolumn{2}{|c|}{ Errors in Estimates } \\
\hline & Position & Velocity & Meas. & Position & Velocity & Position & Velocity \\
\hline $\mathbf{T}=\mathbf{K t}$ & $\mathbf{X}_{1}$ & $\mathbf{X}_{2}$ & $\mathbf{Z}(\mathbf{k})$ & $\mathbf{X}_{1}(\mathbf{K})$ & $\mathbf{X}_{\mathbf{2}}(\mathbf{k})$ & $\mathbf{P}_{11}(\mathbf{k})$ & $\mathbf{P}_{22}(\mathbf{k})$ \\
\hline $\mathbf{0}$ & 100 & $\mathbf{0}$ & & 95 & 1 & 10 & 1 \\
\hline 1 & 99.5 & -1 & 100 & 99.6 & 0.4 & 0.9 & 0.9 \\
\hline 2 & 97.0 & -2.4 & 96.4 & 97.2 & -2.1 & 0.7 & 0.6 \\
\hline 3 & 95.0 & -3.2 & 94.0 & 94.8 & $-\mathbf{3 . 0}$ & 0.7 & 0.3 \\
\hline 4 & 92.0 & -4 & 92.7 & 92.4 & -3.7 & 0.6 & 0.2 \\
\hline 5 & 89.0 & -4.8 & 89.2 & 89.4 & -4.6 & 0.5 & 0.1 \\
\hline
\end{tabular}

VI.

CONCLUSION

Kalman Filters are implemented to find estimates of the position of an attacking missile. Results have shown that the estimated values are very close to the measured values when the object is close to the target. 


\section{REFERENCES}

[1] Bayesian state estimation for tracking and guidance using the bootstrap filter, N Gordon, D Salmond, C Ewing, Jornal of Guidance, Control, and Dynamics, vol. 18, No. 6, Nov. - Dec. 1995.

[2] Tutorial on Particle Filters for OnlineNonlinear/Non-Gaussian Bayesian Tracking M. Sanjeev Arulampalam, Simon Maskell, Neil Gordon, and Tim Clapp.

[3] Kalman Filter - Wikipedia - the free encyclopedia, march, 2013.

[4] Understanding and Applying Kalman Filtering, Lindsay Kleeman, Department of Electrical and Computer Systems Engineering, Monash University, Clayton. 